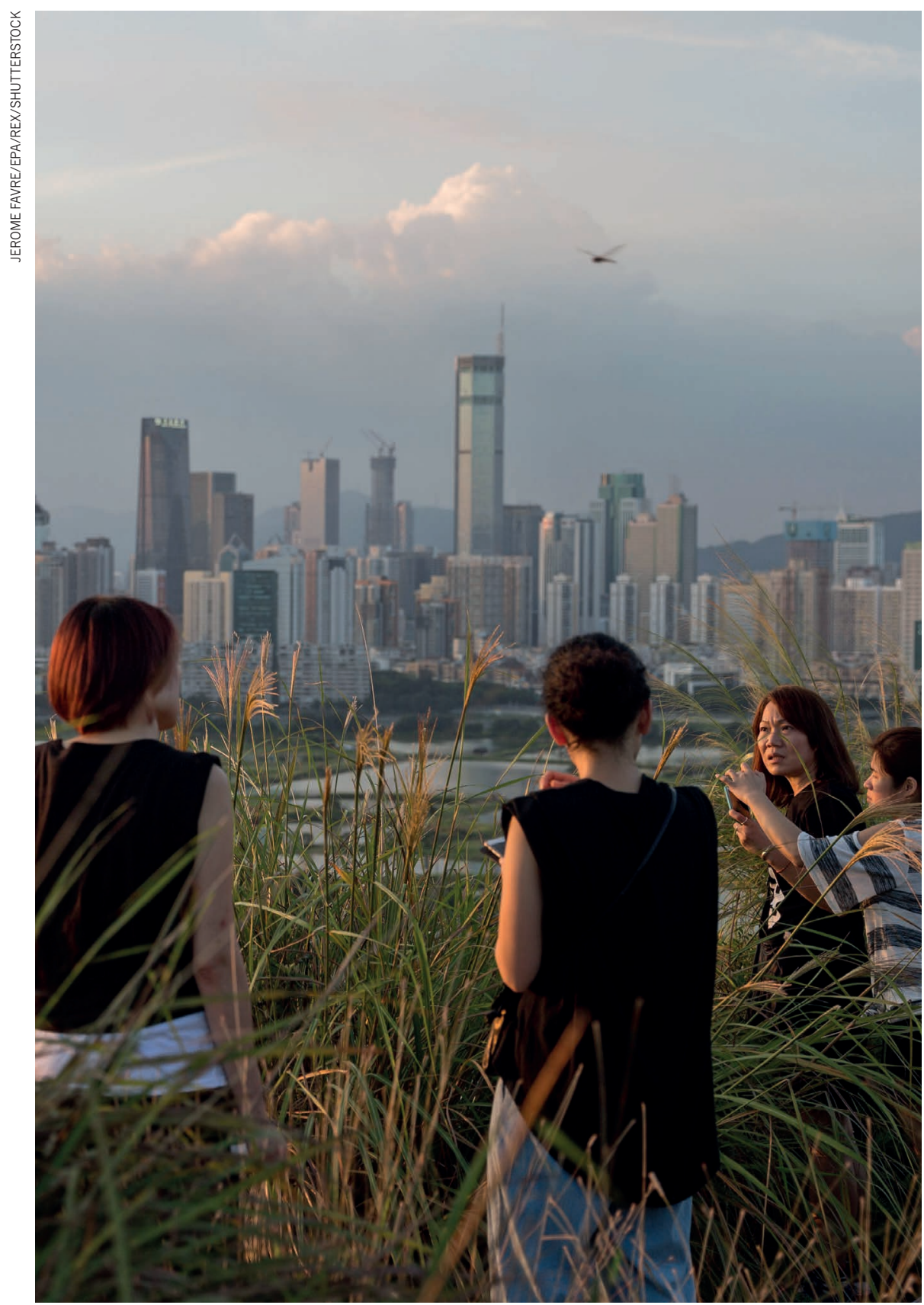

Historically an unremarkable fishing port, Shenzhen is now the richest major city in the country per capita, and China's most innovative metropolis.
$\mathrm{W}$ hen it comes to basic research, Shenzhen has long been the little brother of neighbouring Guangzhou. The two cities are located 130 kilometres apart at the mouth of the Pearl River in China's richest province, Guangdong.

While Guangzhou's universities and public research institutes draw researchers and students from across the country, Shenzhen attracts technology and telecommunications titans such as Tencent and Huawei. But that dichotomy is beginning to change.

In 1980, the central government chose the small fishing town of Shenzhen to be the country's first special economic zone, which welcomed foreign and domestic investment without requiring the government's explicit approval. By 2016, Shenzhen's high-tech industries produced RMB784.7 billion (US $\$ 116.8$ billion) in revenue, accounting for $40 \%$ of the city's GDP. Just over $4.3 \%$ of its GDP went to R\&D, the highest percentage after Beijing, amounting to more than RMB84 billion (US\$12.6 billion).

With 11 million residents, Shenzhen is now the richest major city in the country per capita. It is also China's most innovative metropolis. Huawei, for example, is the only Chinese company ranked in Clarivate Analytics Top 100 Global Innovators, and DJI Innovations Science and Technology has become the world's largest manufacturer of civilian drones. Both companies are based in Shenzhen.

"Shenzhen's market-driven economy and friendly environment has attracted innovative entrepreneurs, but, for a long time, it lacked local science and technology resources and talent," says Du Debin. Until about a decade ago Shenzhen had only one multidisciplinary university involved in research - Shenzhen University (SZU). And as it stands, SZU has not been recognized by the Ministry of Education as a national key university, which would give it extra funding and the pick of the country's top students. But in the past ten years, Shenzhen's academic research landscape has begun to gain ground.

\section{FRIENDLY ADVERSARY}

Guangzhou, the capital of Guangdong Province, has been at the centre of international trade since the 18th century. Guangzhou is home to most of the universities, professors and public research institutions in Guangdong.

In 2016, universities in Guangzhou took in more than one million undergraduate students - more than any other city in China, and 11 times more than Shenzhen. Sun Yat-sen University (SYSU) and South China University of Technology (SCUT) are among the top 50 institutions in China in the Nature Index. In 2016, Guangzhou had a weighted fractional count (WFC) of 228, more than double that of Shenzhen at 103.

However Guangzhou's R\&D expenditure in 2016 - RMB45.7 billion (US $\$ 6.9$ billion) accounted for only $2.3 \%$ of its GDP. That's about 


\begin{tabular}{|c|c|c|}
\hline & Guangzhou & Shenzhen \\
\hline Population $^{1}$ & $14 m$ & $11.91 \mathrm{~m}$ \\
\hline GDP per capita ${ }^{1}$ & US\$21,200 & US\$25,400 \\
\hline R\&D as $\%$ of GDP 2 & $2.3 \%$ & $4.3 \%$ \\
\hline R\&D personnel ${ }^{3}$ & 94,200 & 178,000 \\
\hline Patents granted ${ }^{4}$ & 161 & 2,900 \\
\hline
\end{tabular}

\section{SHARE OF OUTPUT OVER TIME}

City-level contribution to the share of authorship in the Nature Index, measured by the share of weighted fractional count (WFC) for that year, compared to China's share.

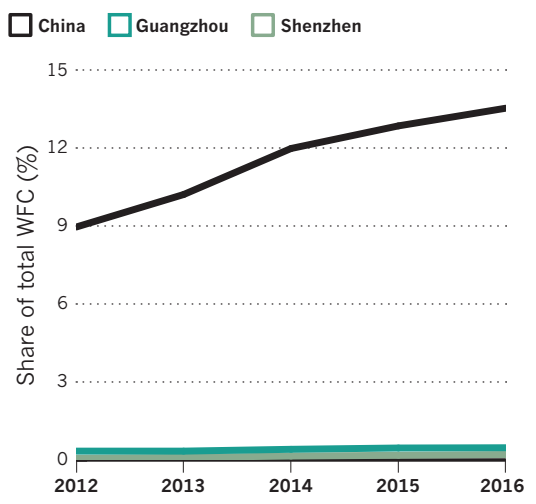

\section{SUBJECT STRENGTHS}

Guangzhou and Shenzhen contribute more to chemistry papers in the Nature Index than any other subject in the natural sciences.

China $\square$ Guangzhou $\square$ Shenzhen

Output is based on WFC 2016

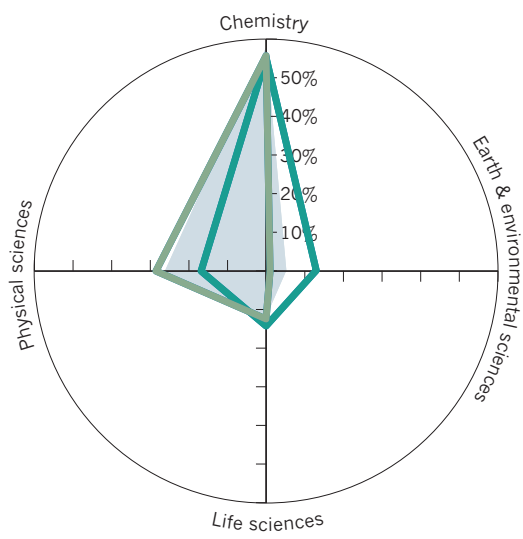

1. Municipal Bureau of Statistics for each city (2016) . Guangdong Provincial Bureau of Statistics (2016) ce and Technology Management 17) (2014 data)

4. United States Patent and Trademark Office (2016) half of Shenzhen's R\&D intensity.

"For decades, Guangzhou has been an important talent supplier to Shenzhen," says Wan Lu, an economist at Guangdong Academy of Social Sciences.

It also offers a constant stream of ideas. Investors based in Shenzhen, home to ChiNext, the Chinese version of the NASDAQ stock market, often invest in inventions and technologies developed by Guangzhou-based institutes.

For example, Shenzhen Capital Group bankrolled the mobile app start-up Mob.com, whose founders came from SCUT. Launched in 2012, Mob.com's services have been used on more than a billion devices worldwide. The citypair's strongest co-authorship link in the Nature Index in 2016 was between the optical components company Shenzhen Star Optoelectronic Technology and SYSU in Guangzhou.

"We often collaborate with Guangzhou colleagues, because their academic disciplines and research devices are much more comprehensive than those available in Shenzhen," says Wu Xuli, a medical scientist at SZU College of Medicine. Guangzhou's medical institutes also offer a much larger database of patient information, he adds.

But Shenzhen has its own benefits. Scientists face less competition for grants, which are often larger. A typical grant in medical science, says Wu, is about RMB300,000 (US\$45,000) in Shenzhen, compared to RMB100,000 (US\$15,000) in Guangzhou.

\section{GROWING UP}

Shenzhen is beginning to come of age. In the last decade, researchers at its institutions have published dozens of papers in Nature, Science, Cell and Physics Review Letters, largely co-authored by researchers in the private sector. A major contributor to these papers has been the company BGI, a CAS spinoff and once the world's largest DNA sequencing provider for research.

"Although Shenzhen does not have many top universities, the pursuit of scientific innovation and market economy, together with the Shenzhen government's beneficial policies, attracted BGI to settle here in 2007," says Liu Xin, vice director of BGI Research, referring to the generous tax exemptions and low-cost land offered to high-tech firms. BGI's achievements include publishing a complete sequence of the giant panda, seahorse and carrot genomes, and conducting large-scale population analyses of the genetic basis of disease.

Physicists in Shenzhen have also benefited from their close proximity to the neutrino observation lab at the Daya Bay nuclear power plant, co-authoring a paper in Physical Review Letters in 2012, which found that neutrinos could oscillate from one state of mass to another to an unexpectedly large degree.

The Shenzhen municipal government has begun to use its financial clout to expand its research infrastructure. Between 2015 and 2016, it signed agreements with 10 universities, including SYSU, SCUT, Tsinghua University,
Peking University, and Harbin Institute of Technology, to either set up campuses in the city or upgrade their original teaching-focused graduate schools to independent campuses that foster research. The city offered the universities free land and financial subsidies for construction and salaries.

In 2009, Shenzhen established the research university Southern University of Science and Technology (SUSTech), which has promised to provide salaries equivalent to those in Western universities, with a minimum research start-up fund of US\$1.5 million over three years.

\section{BILATERAL COLLABORATIONS}

The number of bilateral partnerships between an institution in Guangzhou and an institution in Shenzhen has increased since 2012.

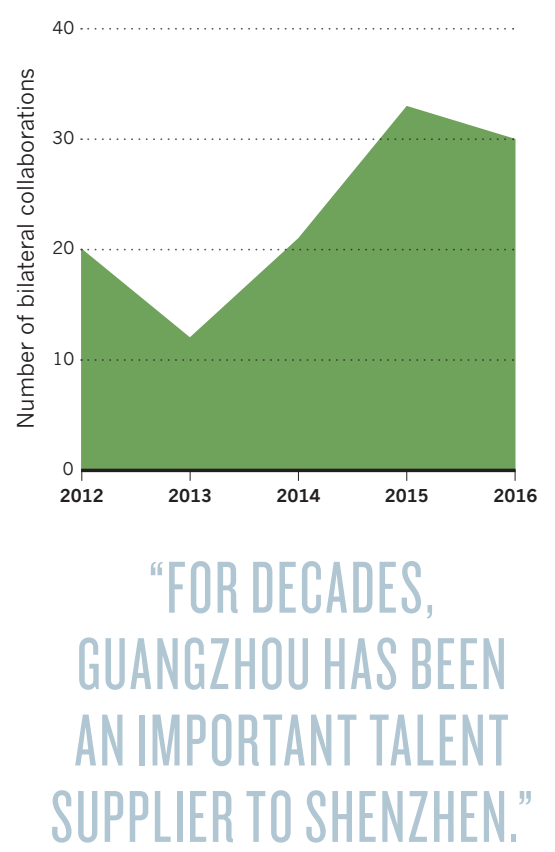

A 2012 study of 28 countries found that public universities in China paid on average one-tenth of the salary of those in Canada, the highest-paying country. "The establishment of these new university campuses will provide new fuel for Shenzhen's innovation," says Du. Within a short period of time, SUSTech has become Shenzhen's top-contributing institute to papers included in the Nature Index - its WFC has shot up from less than 1 in 2012 to 27 in 2016.

Not to be left behind, Guangzhou has also vowed to increase its $\mathrm{R} \& \mathrm{D}$ expenditure to $3 \%$ of GDP by 2020 , as reported in its 13 th fiveyear plan, released in 2016. Since 2015, it has worked with Shenzhen to build a GuangzhouShenzhen Science, Technology and Innovation Corridor, which aims to replicate Boston's Route 128. The corridor will integrate Guangzhou's academic research capacities with Shenzhen's technology development infrastructure, and manufacturing bases in industrial cities between the two municipalities. 


\section{TOP 10 INSTITUTIONS IN BEIJING}

\begin{tabular}{|c|c|c|c|c|c|}
\hline RANK & INSTITUTION & WFC 2012 & WFC 2016 & $\begin{array}{l}\text { CHANGE IN WFC } \\
2012-2016\end{array}$ & AC 2016 \\
\hline 2 & Tsinghua University & 178.6 & 252.2 & $41 \%$ & 841 \\
\hline 4 & Institute of Chemistry, CAS & 112.5 & 124.1 & $10 \%$ & 382 \\
\hline 5 & Institute of Physics, CAS & 81.3 & 76.1 & $-6 \%$ & 294 \\
\hline 8 & People's Liberation Army & 31.9 & 42.4 & $33 \%$ & 161 \\
\hline 9 & National Center for Nanoscience and Technology, CAS & 24.9 & 38.4 & $54 \%$ & 121 \\
\hline 10 & Technical Institute of Physics and Chemistry, CAS & 26.5 & 36.7 & $39 \%$ & 81 \\
\hline
\end{tabular}

\section{TOP 10 INSTITUTIONS IN SHANGHAI}

\begin{tabular}{|c|c|c|c|c|c|}
\hline RANK & INSTITUTION & WFC 2012 & WFC 2016 & $\begin{array}{c}\text { CHANGE IN WFC } \\
2012-2016\end{array}$ & AC 2016 \\
\hline 1 & Fudan University & 121.5 & 137.6 & $13 \%$ & 363 \\
\hline 2 & Shanghai Institute of Organic Chemistry, CAS & 103.9 & 103.8 & $0 \%$ & 230 \\
\hline 3 & Shanghai Jiao Tong University & 80.0 & 93.4 & $17 \%$ & 424 \\
\hline 4 & East China University of Science and Technology & 67.3 & 79.6 & $18 \%$ & 168 \\
\hline 5 & East China Normal University & 35.5 & 71.8 & $102 \%$ & 149 \\
\hline 6 & Shanghai Institutes for Biological Sciences, CAS & 45.7 & 58.8 & $29 \%$ & 167 \\
\hline 7 & Tongji University & 20.8 & 26.2 & $26 \%$ & 89 \\
\hline 8 & Shanghai Institute of Materia Medica, CAS & 19.9 & 24.7 & $24 \%$ & 62 \\
\hline 9 & Shanghai University & 21.9 & 24.3 & $11 \%$ & 74 \\
\hline 10 & Shanghai Institute of Applied Physics, CAS & 17.3 & 16.8 & $-3 \%$ & 57 \\
\hline
\end{tabular}

\section{TOP 10 INSTITUTIONS IN GUANGZHOU}

\begin{tabular}{|c|c|c|c|c|c|}
\hline RANK & INSTITUTION & WFC 2012 & WFC 2016 & $\begin{array}{c}\text { CHANGE IN WFC } \\
2012-2016\end{array}$ & AC 2016 \\
\hline 1 & Sun Yat-sen University & 80.1 & 90.8 & $13 \%$ & 271 \\
\hline 2 & South China University of Technology & 31.4 & 56.4 & $79 \%$ & 105 \\
\hline 3 & South China Sea Institute of Oceanology, CAS & 10.4 & 15.9 & $52 \%$ & 38 \\
\hline 4 & Jinan University & 10.0 & 12.3 & $23 \%$ & 44 \\
\hline 5 & South China Normal University & 12.1 & 11.8 & $-3 \%$ & 25 \\
\hline 6 & Guangzhou Institute of Geochemistry, CAS & 3.8 & 8.0 & $108 \%$ & 17 \\
\hline 7 & Southern Medical University & 1.8 & 6.1 & $238 \%$ & 19 \\
\hline 8 & Guangzhou Institutes of Biomedicine and Health, CAS & 14.3 & 5.1 & $-64 \%$ & 18 \\
\hline 9 & Guangzhou Medical University & 1.2 & 5.0 & $304 \%$ & 24 \\
\hline 10 & South China Agricultural University & 1.7 & 3.5 & $107 \%$ & 15 \\
\hline
\end{tabular}

\section{TOP 10 INSTITUTIONS IN SHENZHEN}

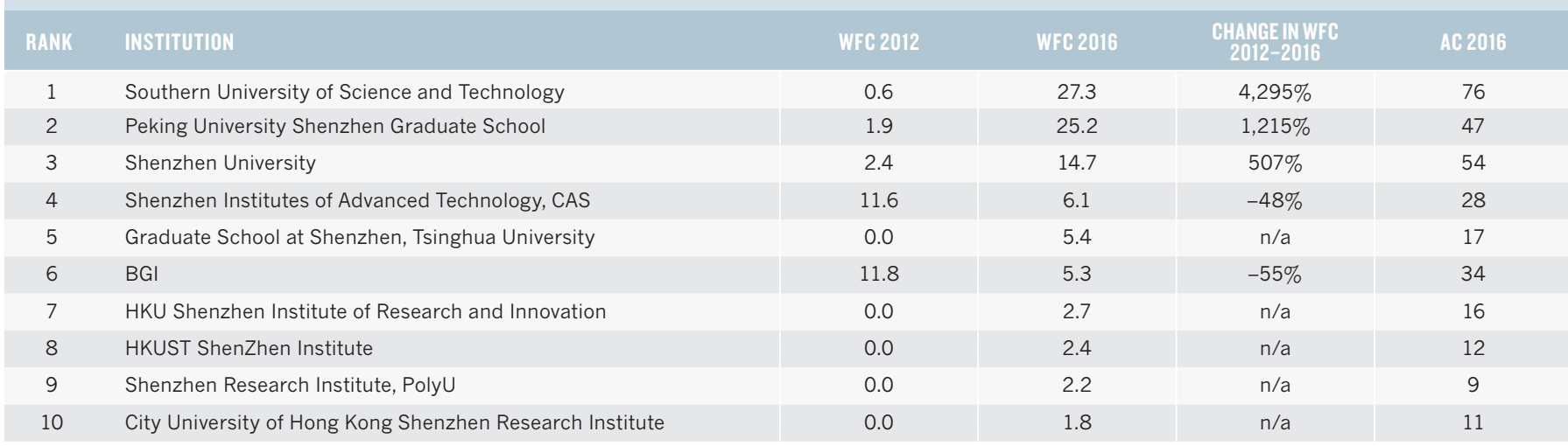




\section{TOP 10 COLLABORATIONS IN BEIJING}

\begin{tabular}{|c|c|c|c|c|c|}
\hline RANK & INSTITUTION 1 & CS 1 & INSTITUTION 2 & CS 2 & TOTAL CS \\
\hline 2 & Peking University & 50.42 & Tsinghua University & 44.84 & 95.26 \\
\hline 4 & University of Chinese Academy of Sciences & 22.55 & Institute of Chemistry, CAS & 46.99 & 69.54 \\
\hline 5 & Tsinghua University & 28.11 & Institute of Physics, CAS & 35.51 & 63.61 \\
\hline 6 & University of Chinese Academy of Sciences & 13.60 & National Astronomical Observatories, CAS & 32.06 & 45.66 \\
\hline 8 & Peking University & 11.73 & National Astronomical Observatories, CAS & 14.10 & 25.83 \\
\hline 9 & University of Chinese Academy of Sciences & 8.76 & Institute of Biophysics, CAS & 16.43 & 25.19 \\
\hline 10 & Beijing Normal University & 14.89 & Peking University & 10.10 & 24.99 \\
\hline
\end{tabular}

\section{TOP 10 COLLABORATIONS IN SHANGHAI}

\begin{tabular}{|c|c|c|c|c|c|}
\hline RANK & INSTITUTION 1 & CS 1 & INSTITUTION 2 & CS 2 & TOTAL GS \\
\hline 1 & Fudan University & 22.11 & Shanghai Jiao Tong University & 6.24 & 28.35 \\
\hline 2 & Shanghai Jiao Tong University & 8.96 & Shanghai Institutes for Biological Sciences, CAS & 6.70 & 15.66 \\
\hline 3 & East China Normal University & 10.79 & Shanghai Institute of Organic Chemistry, CAS & 2.74 & 13.53 \\
\hline 4 & East China University of Science and Technology & 6.66 & Shanghai Institute of Organic Chemistry, CAS & 4.51 & 11.17 \\
\hline 5 & ShanghaiTech University & 1.15 & Shanghai Institutes for Biological Sciences, CAS & 9.81 & 10.96 \\
\hline 6 & Fudan University & 5.00 & Shanghai Institutes for Biological Sciences, CAS & 5.25 & 10.24 \\
\hline 7 & Fudan University & 4.39 & Shanghai Institute of Organic Chemistry, CAS & 4.86 & 9.25 \\
\hline 8 & East China University of Science and Technology & 4.73 & Shanghai Jiao Tong University & 4.04 & 8.77 \\
\hline 9 & University of Chinese Academy of Sciences, Shanghai & 2.26 & Shanghai Institutes for Biological Sciences, CAS & 6.41 & 8.67 \\
\hline 10 & East China University of Science and Technology & 1.24 & Shanghai Institutes for Biological Sciences, CAS & 6.55 & 7.78 \\
\hline
\end{tabular}

\section{TOP 10 COLLABORATIONS IN GUANGZHOU}

\begin{tabular}{|c|l|l|}
\hline RANK & INSTITUTION 1 & CS1 \\
\hline 1 & Guangzhou Medical University & 2.26 \\
\hline 2 & South China University of Technology & 2.31 \\
\hline 3 & Guangzhou Medical University & 0.45 \\
\hline 4 & Jinan University & 0.73 \\
\hline 5 & Sun Yat-sen University & 1.48 \\
\hline 6 & South China Normal University & 0.60 \\
\hline 7 & South China Agricultural University & 0.49 \\
\hline 8 & Guangzhou Medical University & 0.04 \\
\hline 8 & Guangzhou University of Chinese Medicine & 0.14 \\
\hline 8 & South China University of Technology & 0.83 \\
\hline 8 & Sun Yat-sen University & 0.88 \\
\hline 9 & Jinan University & 0.25 \\
\hline 10 & Jinan University & 0.64 \\
\hline
\end{tabular}

INSTITUTION 2

Guangzhou Institutes of Biomedicine and Health, CAS

Sun Yat-sen University

Sun Yat-sen University

Sun Yat-sen University

Guangzhou Institutes of Biomedicine and Health, CAS

Sun Yat-sen University

Sun Yat-sen University

Southern Medical University

South China Agricultural University

Guangdong Provincial Academy of Chinese Medical Sciences, CACMS

Guangzhou Institute of Geochemistry, CAS

South China Sea Institute of Oceanology, CAS

Guangzhou Institutes of Biomedicine and Health, CAS
CS2

TOTAL CS

2.21

1.94

2.47

1.70

0.34

1.10

1.10

0.96

0.86

0.17

0.13

0.67

0.27

4.46
4.24
2.92
2.44
1.82
1.70
1.59
1.00
1.00
1.00
1.00
0.92
0.91

\section{TOP 10 COLLABORATIONS IN SHENZHEN}

\begin{tabular}{|c|c|c|c|c|c|}
\hline RANK & INSTITUTION 1 & CS 1 & INSTITUTION 2 & CS2 & TOTAL CS \\
\hline 2 & Graduate School at Shenzhen, Tsinghua University & 0.31 & Peking University Shenzhen Graduate School & 1.21 & 1.52 \\
\hline 4 & Southern University of Science and Technology & 0.61 & Longgang Central Hospital, Shenzhen & 0.22 & 0.83 \\
\hline 5 & Peking University Shenzhen Graduate School & 0.18 & Shenzhen Institutes of Advanced Technology, CAS & 0.59 & 0.77 \\
\hline 6 & HKUST ShenZhen Institute & 0.25 & Peking University Shenzhen Graduate School & 0.50 & 0.75 \\
\hline 9 & Peking University Shenzhen Graduate School & 0.61 & Shenzhen University & 0.04 & 0.64 \\
\hline 10 & Graduate School at Shenzhen, Tsinghua University0 & 0.50 & Shenzhen South China Pharmaceutical Co., Ltd & 0.11 & 0.61 \\
\hline
\end{tabular}

